\title{
Lymphnode cytopathology and CD4 count in HIV positive patients
}

\author{
Amrutha Gorva ${ }^{1}$, Saritha Karre ${ }^{2, *}$, Chandrakumar Shanmugam ${ }^{3}$, Satyanarayana \\ Veeragandham ${ }^{4}$, V D Praveenkumar Gorrela ${ }^{5}$ \\ ${ }^{\mathbf{1}}$ Assistant Profesor, Dept. of Pathology, Mandya Institute of Medical Sciences, Mandya, ${ }^{\mathbf{2}}$ Assistant Professor, \\ ${ }^{3}$ Associate Professor \& I/C HOD, ${ }^{5}$ Junior Resident, Dept. of Pathology, ESIC Medical College, Sanathnagar, \\ Hyderabad, ${ }^{4} \mathrm{HOD}$, Dept. of Pathology, Kamineni Institute of Medical Sciences, Narketpally, Nalgonda, \\ Telangana, India
}

*Corresponding Author:

Email: dr_saritha14@yahoo.co.in

\begin{abstract}
Introduction: CD4 is an important prognostic as well as therapeutic marker in the management of HIV and this study identifies the correlation between CD4 counts and FNAC lymphnode (LN) patterns in these patients.

Study Design: The study was conducted in the rural areas at Nalgonda district by Kamineni Institute of Medical Sciences, Telangana. Eventhough Fine Needle Aspiration Cytology (FNAC) was done on total 56 selected patients who had lymphadenopathy and were tested HIV positive, only 50 of them have CD4 counts and were selected as final study group. Suspected cytosmears have acid fast staining done by Ziehl Nelson technique. The final cytopathological findings were correlated with CD4 counts.

Results: In our study, high CD4 counts were associated with reactive lymphadenitis $(26 \% ; 13 / 50)$ most and Low CD4 counts were associated with less frequent suppurative lymphadenitis and particulary TB lymphadenitis was noticed in 56\% (28/50) and morphologically exhibited 4 different patterns- caseous with epitheloid granuloma, only caseous necrosis, only epitheloid granuloma and suppurative.

Conclusion: CD4 count correlated well with the different stages of immunosuppression in HIV patients. The patients with reactive lymphadenitis demonstrated high CD4 counts suggesting good immunological response. The low CD4 counts seen in patients with suppurative lymphadenitis and cryptococcal infection suggests severe immuno-compromised state.
\end{abstract}

Keywords: Cytopathology, CD4 count, Granuloma, HIV, Lymphnode, Tuberculosis.

Received: $19^{\text {th }}$ September, 2017

\section{Introduction}

Human immunodeficiency virus $^{1}$ (HIV) infection causes acquired immunodeficiency predisposing the affected patients to opportunitistic infections and secondary cancers, sometimes involving multiple systems. ${ }^{2}$ As per UNAIDS report on Global AIDS epidemic 2010, people living with HIV at the end of 2011 were around 34 million and major burden was shared among south Africa, Nigeria and India.

Pathogenetically, HIV infects T cells using CD4 molecule as receptor and CCR5 \& CXCR4 as co-receptors. Once infected the CD4 cells are destroyed by lysis due to cytopathic effect. The CD4 count dwindles as the disease progresses to final stage AIDS as uninfected cells also undergo apoptosis. $^{3}$

The role of CD4 counts in predicting immunological damage during infection and monitoring recovery after antiretroviral treatment has been well established and the reason behind considering CD4 counts along with different clinical spectrum of HIV infection in the current CDC classification system for HIV infected adolescents and adults. ${ }^{4}$
Accepted: $11^{\text {th }}$ October, 2017

One of the earliest manifestation of HIV infection is lymphadenapathy. ${ }^{5}$ In India, there is need for simple and cost effective investigation for HIV associated lymphadenopathy and FNAC, an outpatient technique suits the best in this regard. The present study describes the cytopathological patterns associated with HIV lymphadenopathy and also demonstrates its correlation with CD4 count.

\section{Materials and Methods}

The present study was conducted by KIMS, Narketpally, Nalgonda after the due approval of Ethical committee. We collected total 56 patients for around 2 years who had lymphadenopathy and tested HIV positive. The NACO (National AIDS Control Organization) guidelines were strictly followed in making HIV diagnosis.

The study group includes only 50 patients with their CD4 counts done. Patients on highly active anti-retroviral treatment (HAART) and Anti Tubercular treatment that could influence FNAC findings were excluded. FNAC was performed with informed consent of the patients 
and after proper clinical evaluation with respect to size and multiplicity of lymphnodes. $22 \mathrm{G}$ disposable needles with cameco syringe holders were used. The collected aspirate was smeared, air dried and fixed with $95 \%$ ethyl alcohol and then stained with routine MGG (May Grunwald Giemsa), H\&E (Haematoxylin-Eosin), PAP (Papanicolaou) techniques. Special staining techniques like AFB (Acid Fast Bacilli) and PAS (Periodic acid-Schiff stain) were done when indicated.

The CDC guidelines of 1985 were followed in reporting Ziehl Nelsen stained smears. Fungal culture was also done. Fluorescent Activated Cell Sorter (FACS) counters were used to calculate the CD4 counts. ANOVA (Analysis of Variation) and Post hoc tests were used for statistical analysis and significance testing.

\section{Results}

Out of 56 HIV patients who were selected for the present study, only 50 cases had both FNAC and CD4 count done. These patients FNAC reports were correlated with CD4 counts based on their age, sex and site distribution. Patients age ranged from 11-70 years, majority (44\%) of patients were young adults (21-30 years) with a male preponderance (males $(35 / 50$, $70 \%$ ) and females $(15 / 50,30 \%)$. Most common clinical presentation was cervical lymphadenopathy alone 46(92\%) and cervical lymphadenopathy with axillary lmphadenopathy was seen in $2(4 \%)$. One $(2 \%)$ patient had presented with bilateral inguinal lymphadenopathy and Generalised lymphadenopathy was seen in $1(2 \%)$ patient.

The cytological findings observed are given in Table 1, the majority of patients demonstrated Tuberculous (TB) lymphadenitis $(56 \%)$ followed by reactive lymphadenitis (26\%). TB Lymphadenitis presented with four morphological pattern (Table 2). The different patterns observed were caseous necrosis alone $4(14.29 \%)$, caseous necrosis along with epitheloid granulomas $21(75 \%)$ and few with Langhan's giant cell, epitheloid granulomas without caseation $2(7.14 \%)$ and suppurative lesion $1(3.57 \%)$. Overall, caseous necrosis seen in 25 out of 28 patients.

The AFB positive staining was noticed in only 6 patients with caseous necrosis with epitheloid cell granulomas, 3 patients with caseous necrosis alone, 2 patients with epithelioid cell granulomas without caseous necrosis. Culture positivity was seen in one patient with AFB negative for bacilli on $\mathrm{ZN}$ stain. One patient with suppuration exhibited strong AFB positivity and all patients with epitheloid cell granuloma without caseation were negative for AFB.

Reactive lymphadenitis characterized by the presence of small lymphocytes, transforming lymphocytes, centrocytes, tingible body macrophages and few histiocytes was the second most common finding in our study. Granulomatous lymphadenitis with epitheloid formation were $6(12 \%)$ and AFB negative were specifically categorized as non-specific granulomatous lymphadenitis. Suppuration and cryptococcal lymphadenitis were $4 \%$ and $2 \%$ with 2 and 1 patient respectively.

The mean CD4 count was calculated for all the patients. Patients with reactive lymphadenits had highest CD4 counts of 348.63 cells/ $\mu 1$ followed by non-specific granulomatous lymphadenitis $(341.33$ cells $/ \mu 1)$. The lowest CD4 count of 74 cells $/ \mu 1$ was observed in Cryptococcus infection. Within TB lymphadenitis patients with suppurative tuberculous lymphadenits demonstrated lowest CD4 counts (84 cells/ $\mu 1)$ followed by only caseous necrosis with mean CD4 of 99 cells/ $\mu 1$. (Table 3) In addition, tuberculous lymphadenits patients were categorized into AFB positive and AFB negative categories and were correlated with CD4 count (Table 3), The mean CD4 count was low for the patients categorized as AFB positive when compared to AFB negative patients $(109.67 \pm 17.97$ cells $/ \mu 1$ vs $230.63 \pm 63.72$ cells/ $\mu 1$ ) respectively. (Table 3 ) This difference of CD4 counts amongst the two categories was statistically significant (pvalue, $<0.0001$ ) which suggests low levels of immunity in AFB positive patients.

Table 1: HIV positive patients exhibiting cytological pattern of lymph nodes (N-50)

\begin{tabular}{|l|c|}
\hline \multicolumn{1}{|c|}{ Diagnosis } & Number of cases \\
\hline Lymphadenitis with TB & $28(56 \%)$ \\
\hline Lymphadenitis with granuloma & $6(12 \%)$ \\
\hline Reactive lymphadenitis & $13(26 \%)$ \\
\hline Lymphadenitis with Acute suppuration & $2(4 \%)$ \\
\hline Cryptococcus & $1(2 \%)$ \\
\hline
\end{tabular}


Table 2: Tuberculous Lymphadenitis with its cytological pattern $(\mathrm{N}=28)$

\begin{tabular}{|l|c|}
\hline \multicolumn{1}{|c|}{ Diagnosis } & Number of cases \\
\hline Epithelioid granuloma with caseous necrosis & $21(75 \%)$ \\
\hline Caseous necrosis & $4(14.29 \%)$ \\
\hline Epithelioid granuloma & $2(7.14 \%)$ \\
\hline Suppurative & $1(3.57 \%)$ \\
\hline
\end{tabular}

Table 3: Correlation between Cytological findings and mean CD4 count

\begin{tabular}{|l|l|c|}
\hline & \multicolumn{1}{|c|}{ Cytology Diagnosis } & Mean CD4 count\& SD \\
\hline 1. & TB Lymphadenitis (AFB +ve) & $109.67 \pm 17.97$ \\
\hline 2. & TB lymphadenitis (AFB -ve) & $230.63 \pm 63.72$ \\
\hline 3. & Granulomatous lymphadenitis & $341.3 \pm 33.3$ \\
\hline 4. & Reactive lymphadenitis & $348.63 \pm 58.6$ \\
\hline 5. & Acute suppurative lymphadenitis & 113 \\
\hline 6. & Cryptococcus & 74 \\
\hline
\end{tabular}
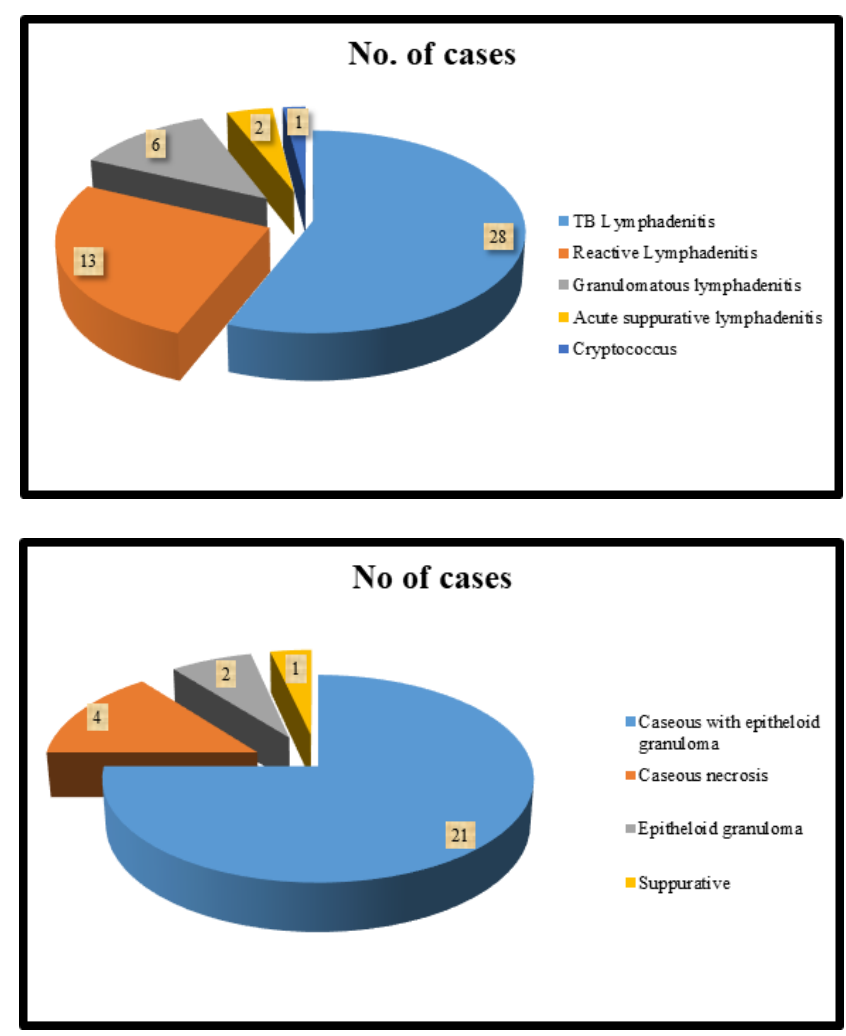

Table 4: Tuberculous lymphadenitis cytological pattern and mean CD4 count correlation

\begin{tabular}{|l|c|c|}
\hline \multicolumn{1}{|c|}{ Cytological Study } & $\begin{array}{c}\text { Number of } \\
\text { cases }\end{array}$ & Mean CD4 count \\
\hline Only caseous necrosis & $4(14.29 \%)$ & 99 \\
\hline $\begin{array}{l}\text { Epithelioid granuloma with } \\
\text { caseous necrosis }\end{array}$ & $21(75 \%)$ & 181.86 \\
\hline Only epithelioid granuloma & $2(7.14 \%)$ & 353.5 \\
\hline Suppurative & $1(3.57 \%)$ & 84 \\
\hline
\end{tabular}

Table 5: Correlation between cytological findings and mean CD4 count in present and other study

\begin{tabular}{|l|c|c|}
\hline Cytology Diagnosis & \multicolumn{2}{|c|}{ Mean CD4 count } \\
\hline & Ratan.K. et al & Present Study \\
\hline TB lymphadenitis & $164.78+\_117.20$ & $170.15+\_40.85$ \\
\hline
\end{tabular}




\begin{tabular}{|l|c|c|}
\hline Granulomatous lymphadenitis & $155.86+\_58.67$ & $341.33+\_33.3$ \\
\hline Reactive lymphadenitis & $328.04+\_123.17$ & $348.63+\_58.6$ \\
\hline Acute suppurative lymphadenitis & $189.71+\_50.71$ & 113 \\
\hline Cryptococcus & $179.91+\_58.67$ & 74 \\
\hline
\end{tabular}

Table 6: Tuberculous lymphadenitis pattern and CD4 count correlation of present and other study

\begin{tabular}{|l|c|c|c|c|}
\hline Cytology pattern & \multicolumn{2}{|c|}{ Guru etal } & \multicolumn{2}{c|}{ Present study } \\
\hline & $\begin{array}{l}\text { Number } \\
\text { of cases }\end{array}$ & Mean CD4 & $\begin{array}{l}\text { Number } \\
\text { of cases }\end{array}$ & Mean CD4 \\
\hline $\begin{array}{l}\text { Only caseous } \\
\text { necrosis }\end{array}$ & 44 & 101.40 & 4 & 99 \\
\hline $\begin{array}{l}\text { Epithelioid } \\
\text { granuloma with } \\
\text { caseous necrosis }\end{array}$ & 45 & 110.50 & 21 & 181.86 \\
\hline $\begin{array}{l}\text { Epithelioid } \\
\text { granuloma }\end{array}$ & 3 & 142.50 & 2 & 353.5 \\
\hline
\end{tabular}

\section{Discussion}

Middle aged population from 25 to 44 were the most commonly HIV affected group globally with a pediatric age incidence of $2 \%$. The risk of developing active TB infection is 20-30 times more common in HIV patients. The statistics shows $13 \%$ of TB patients were HIV positive and were 3,60,000 deaths from HIV related TB in 2010 .

HIV affects cell mediated immunity grossly with involvement of various groups of lymphnodes. ${ }^{6}$ FNAC of the affected LN serve as a cost effective, easy to perform, reliable, and quick diagnostic tool in this setting and renders early diagnosis in these patients.

In our study, 92\% showed cervical lymphadenopathy, 2 cases showed axillary along with cervical and one case showed bilateral inguinal lymphadenopathy and one showed generalized lymphadenopathy. These findings of our study are comparable with a study by Guru et $\mathrm{al}^{5}$ that reported cervical lymphadenopathy in $78.76 \%$ and axillary lymphadenopathy $21.23 \%$ in HIV patients.

21-30 years (44\%) were the most commonly affected followed by 30-40 years (42\%) age group in HIV patients, inconcordance with previous studies like (Shenoy etal ${ }^{7}[44 \%$ between 25-30years] \& Vanishri et $\mathrm{al}^{8}$ [44.4\% between 21-30 years of age group]). Male (70\%) preponderance was noted similar to study by Vanishri etal ${ }^{8}(72.22 \%)$.

TB Lymphadenitis (56\%) was the most common followed by reactive lymphadenitis $(28 \%)$ as shown in many previous studies Shenoy et $\mathrm{al}^{7}(50 \%)$ and Jayaram et $\mathrm{al}^{9}(53.84 \%)$ studies. The occurrence of suppurative lymphadenitis $4 \%$ go well in concurrence with other studies (1.3\% to $2 \%) .{ }^{8}$ Cryptococcal infection was encountered in one case $(2 \%)$ and its morphology as seen in other studies. ${ }^{5,9}$

CD4 count was good (high) in patients with reactive lymphadenitis with mean count $348.63 \pm 58.6$ cells $/ \mu 1$ and patients with crytococcal infection had the least CD4 count of 74 cells $/ \mu 1$. The plausible explanation for this could be that low CD4 count indicates pronounced immunosuppression predisposing the HIV patients to opportunistic fungal infections like Cryptococcus. This is in concordance with study by Ratan $\mathrm{K}$ et al. ${ }^{10}$ Amongst the morphological patterns of TB lymphadenitis lowest CD4 count was observed in the suppurative type similar to other studies. ${ }^{11,12}$ Additionally, our study apart from CD4 count correlation with cytopathology, patients with TB lymphadenopathy were further categorized into AFB positive and negative groups. Low mean CD4 counts were associated with AFB positive than negative groups. This observation could be due to HIV patients with low CD4 counts poor mounters of immune response especially against tuberculous bacilli.

\section{Conclusion}

HIV patients with lower CD4 counts and associated AFB positivity are indicative of poor immunological status and during non feasibility of CD4 the positive cytological smear for AFB by $\mathrm{ZN}$ stain can be used as an indirect marker of lower CD4 count especially in the rural setup. Furthermore FNAC correlation with CD4 counts provides insight into immune status and stage of the disease in HIV patients. 


\section{References}

1. Pairkh U.R, Goswami H.M, Nanavati M.G, Bisen V.V, Patel S., Menpara C.B, et al. Diagnostic Utility of FNAC in HIV Positive

Lymphadenopathy. Journal of Clinical Research Letters 2012;3(2):37-40.

2. Février M, Dorgham K, Rebollo A. CD4 T Cell Depletion in Human Immunodeficiency Virus (HIV) Infection: Role of Apoptosis, Viruses 2011;3(5):586-612.

3. Kumar V, Abbas AK, Fauato N, Aster JC. Diseases of the Immune System, Pathologic basis of disease (Philadelphia: Saunders Elsevier) 2010;8:183-258.

4. Herbeuval J.P, Grivel J.C, Boasso A, Hardy A.W, Chougnet C, Dolan M.J, Hideo et al. CD4 T-cell death induced by infectious and noninfectious HIV-1: role of type 1 interferon-dependent, TRAIL/DR5-mediated apoptosis, BLOOD 2005;106(10):3524-31.

5. Kumarguru, M.H.Kulkarni, N.S.Kamakeri. FNAC of peripheral lymphnodes in HIV-positive patients Scientific medicine 2009;1(2).

6. Martin Bates E, Tanner A, Survana SK et al. Use of fine needle aspiration cytology for investigating lymphadenopathy in HIV positive patients. J Clin Pathol 1993;46(6):564-6.

7. Shenoy R, Kapadi SN, Pai KP, Kini H, Mallya S, Khadilkar UN, et al. Fine needle aspiration diagnosis in HIV - related Lymphadenopathy in Mangalore India. Acta Cytol. 2001;46:35-9.

8. Vanisri H R, Nandini N M, Sunila R. Fine needle aspiration cytology finding in human immunodeficiency virus lymphadenopathy, IJPM 2008;51(4):481-4.

9. Jayaram G, Chew MT. Fine needle aspiration cytology of lymph node in HIV - infected individual ActaCytol. 2000;44:960-6.

10. Ratan K, Bhubon Singh N, Arunkumar K, Shikha NG, Niteshor MS. Cytomorphological diagnosis of lymphadenopathy by fine needle aspiration cytology in HIV positive patients and its correlation with CD4 count. A hospital based 2 years cross sectional study. IOSR-JDMS, 2014;13(7):149-54.

11. JosephSushilRao, Jaya Kumari S, Ushakini. Correlation of CD4 counts with the FNAC patterns of Tubercular lymphadenitis in patients with HIV. A cross sectional pilot study. Diagnostic cytopathology 2014;43(1):16-20.

12. A. Shobhana, SK Guha, K Mitra, A Dasgupta, DK Neogi, SC Hgra. People living with HIV infection/AIDS- A study of lymphnode FNAC and CD4 count, IJMM 2002;20(2):99-101. 